\title{
The genome sequence of the ornate tailed digger wasp,
}

\section{Cerceris rybyensis (Linnaeus, 1771) [version 1; peer review: 2}

\section{approved]}

\author{
Liam M. Crowley (iD), \\ University of Oxford and Wytham Woods Genome Acquisition Lab, \\ Darwin Tree of Life Barcoding collective, \\ Wellcome Sanger Institute Tree of Life programme, \\ Wellcome Sanger Institute Scientific Operations: DNA Pipelines collective, \\ Tree of Life Core Informatics collective, Darwin Tree of Life Consortium \\ ${ }^{1}$ Department of Zoology, University of Oxford, Oxford, UK
}

V1 First published: $13 \operatorname{Dec} 2021,6: 337$

https://doi.org/10.12688/wellcomeopenres.17483.1

Latest published: $13 \mathrm{Dec} 2021,6: 337$

https://doi.org/10.12688/wellcomeopenres.17483.1

\section{Abstract}

We present a genome assembly from an individual female Cerceris rybyensis (the ornate tailed digger wasp; Arthropoda; Insecta; Lepidoptera; Noctuidae). The genome sequence is 574 megabases in span. The majority of the assembly, $89.81 \%$, is scaffolded into 14 chromosomal pseudomolecules.

Keywords

Cerceris rybyensis, ornate tailed digger wasp, genome sequence, chromosomal, Hymenoptera

This article is included in the Tree of Life gateway.

\section{Open Peer Review \\ Approval Status \\ 1 \\ 2 \\ version 1 \\ 13 Dec 2021

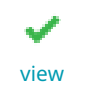 \\ view \\ 1. Peter K Dearden ID, University of Otago, Dunedin, New Zealand \\ 2. Rodolpho S. T. Menezes (D), Universidade Federal de Santa Maria, Santa Maria, RS, Brazil}

Any reports and responses or comments on the article can be found at the end of the article. 
Corresponding author: Darwin Tree of Life Consortium (mark.blaxter@sanger.ac.uk)

Author roles: Crowley LM: Investigation, Resources, Writing - Original Draft Preparation;

Competing interests: No competing interests were disclosed.

Grant information: This work was supported by Wellcome through core funding to the Wellcome Sanger Institute (206194) and the Darwin Tree of Life Discretionary Award (218328).

The funders had no role in study design, data collection and analysis, decision to publish, or preparation of the manuscript.

Copyright: (c) 2021 Crowley LM et al. This is an open access article distributed under the terms of the Creative Commons Attribution License, which permits unrestricted use, distribution, and reproduction in any medium, provided the original work is properly cited.

How to cite this article: Crowley LM, University of Oxford and Wytham Woods Genome Acquisition Lab, Darwin Tree of Life Barcoding collective et al. The genome sequence of the ornate tailed digger wasp, Cerceris rybyensis (Linnaeus, 1771) [version 1; peer review: 2 approved] Wellcome Open Research 2021, 6:337 https://doi.org/10.12688/wellcomeopenres.17483.1

First published: 13 Dec 2021, 6:337 https://doi.org/10.12688/wellcomeopenres.17483.1 


\section{Species taxonomy}

Eukaryota; Metazoa; Ecdysozoa; Arthropoda; Hexapoda; Insecta; Pterygota; Neoptera; Endopterygota; Hymenoptera; Apocrita; Aculeata; Apoidea; Spheciformes; Crabronidae; Philanthinae; Cercerini; Cerceris; Cerceris rybyensis (Linnaeus, 1771) (NCBI:txid1167272).

\section{Background}

The Ornate Tailed Digger Wasp, Cerceris rybyensis, is a widespread, mid-sized $(6-12 \mathrm{~mm})$ digger wasp that occurs throughout the Palearctic. In the UK it is common across southern England in habitats with sandy soil and on chalk grassland. Adults are yellow and black with a distinctively ribbed metasoma characteristic of the Genus, that is irregularly banded black and yellow in this species, distinguishing it from other Cerceris species in the UK.

It is a univoltine species, with a flight period from late June to early September. It often nests in dense aggregations, occasionally intermixed with Cerceris arenaria. Adult females dig a 10-15 $\mathrm{cm}$ deep burrow with multiple cells branching off laterally (Else, 1997). The females hunt small to medium sized bees of several genera, particularly Lasioglossum and Halictus. Often prey returning to their nest fully laden with pollen are favoured, although males and unladen females are also taken. Navigation back to the nest is aided by the undertaking of arcing orientation flights around the nest as they leave (Zeil, 1993). Prey is paralysed by stinging and malaxated just behind the head, before being carried back to a pre-prepared nest cell within the burrow. Paralysis is achieved by the delivery of venom via the stinger, which contains a complex mix of biogenic amines, peptides and proteins that act as toxins, neuromodulators, immunomodulators, metabolic-modulators and antimicrobial agents (Kote et al., 2019). Each cell is stocked with 5-8 paralysed bees, which remain alive for around 2 days (Lomholdt, 1975). A single egg is laid in each cell, which hatches and consumes the prey provisions before pupating. Individuals remain within the pupal cocoon over winter. Adults visit the flowers of various species to feed on nectar, including common hogweed (Heracleum sphondylium), wild carrot (Daucus carota), yarrow (Achillea millefolium) and creeping thistle (Cirsium arvense).

\section{Genome sequence report}

The genome was sequenced from a single female $C$. rybyensis (Figure 1) collected from Wytham Woods, Oxfordshire, UK (latitude 51.782, longitude -1.316). A total of 58-fold coverage in Pacific Biosciences single-molecule, circular consensus ( $\mathrm{HiFi}$ ) long reads and 91-fold coverage in $10 \mathrm{X}$ Genomics read clouds were generated. Primary assembly contigs were scaffolded with chromosome conformation Hi-C data. Manual assembly curation corrected 13 missing/misjoins and removed 1 haplotypic duplication, reducing the scaffold number by $1.47 \%$.

The final assembly has a total length of $574 \mathrm{Mb}$ in 51 sequence scaffolds with a scaffold N50 of $19.0 \mathrm{Mb}$ (Table 1). Of the assembly sequence, $89.81 \%$ was assigned to 14
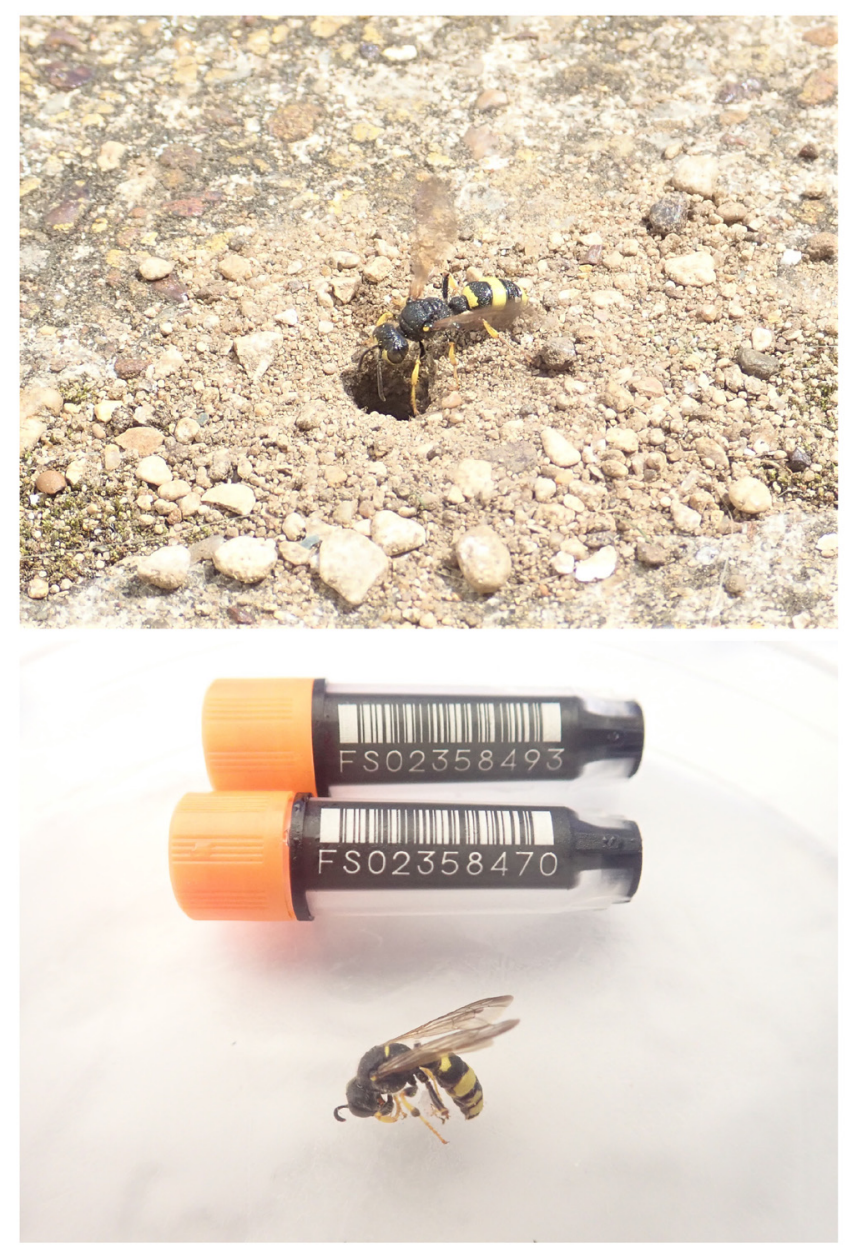

Figure 1. Image of the iyCerRyby1 specimen taken prior to capture (top) and during preservation and processing (bottom).

chromosomal-level scaffolds, representing 14 autosomes (numbered by sequence length) (Figure 2-Figure 5; Table 2). A large number of repetitive scaffolds were produced in sequencing, which were not able to be placed in the assembly. The assembly has a BUSCO v5.1.2 (Manni et al., 2021) completeness of $96.8 \%$ (single $96.6 \%$, duplicated $0.3 \%$ ) using the hymenoptera_odb10 reference set. While not fully phased, the assembly deposited is of one haplotype. Contigs corresponding to the second haplotype have also been deposited.

\section{Methods}

Sample acquisition and DNA extraction

A single female $C$. rybyensis was collected from Wytham Woods, Oxfordshire, UK (latitude 51.772, longitude -1.338) by Liam Crowley, University of Oxford, using a net. The specimen was identified by the same individual and preserved on dry ice prior to transfer to the Wellcome Sanger Institute.

DNA was extracted at the Tree of Life laboratory, Wellcome Sanger Institute. The iyCerRyby1 sample was weighed and dissected on dry ice with tissue set aside for Hi-C sequencing. Abdomen tissue was disrupted using a Nippi Powermasher 
Table 1. Genome data for Cerceris rybyensis, iyCerRyby1.1.

\begin{tabular}{|c|c|}
\hline \multicolumn{2}{|l|}{ Project accession data } \\
\hline Assembly identifier & iyCerRyby1.1 \\
\hline Species & Cerceris rybyensis \\
\hline Specimen & iyCerRyby 1 \\
\hline NCBI taxonomy ID & NCBI:txid1167272 \\
\hline BioProject & PRJEB45199 \\
\hline BioSample ID & SAMEA7701329 \\
\hline Isolate information & Female, abdomen (genome assembly), head/thorax (Hi-C) \\
\hline \multicolumn{2}{|l|}{ Raw data accessions } \\
\hline PacificBiosciences SEQUEL II & ERR6436387 \\
\hline 10X Genomics Illumina & ERR6054972-ERR6054975 \\
\hline Hi-C Illumina & ERR6054976 \\
\hline \multicolumn{2}{|l|}{ Genome assembly } \\
\hline Assembly accession & GCA_910591515.1 \\
\hline Accession of alternate haplotype & GCA_910591445.1 \\
\hline Span (Mb) & 418 \\
\hline Number of contigs & 710 \\
\hline Contig N50 length (Mb) & 15.2 \\
\hline Number of scaffolds & 671 \\
\hline Scaffold N50 length (Mb) & 26.2 \\
\hline Longest scaffold (Mb) & 66.4 \\
\hline BUSCO* genome score & C:96.8\%[S:96.6\%,D:0.3\%],F:0.8\%,M:2.4\%,n:5991 \\
\hline
\end{tabular}

fitted with a BioMasher pestle. Fragment size analysis of 0.01-0.5 ng of DNA was then performed using an Agilent FemtoPulse. High molecular weight (HMW) DNA was extracted using the Qiagen MagAttract HMW DNA extraction kit. Low molecular weight DNA was removed from a 200-ng aliquot of extracted DNA using 0.8X AMpure XP purification kit prior to $10 \mathrm{X}$ Chromium sequencing; a minimum of $50 \mathrm{ng}$ DNA was submitted for $10 \mathrm{X}$ sequencing. HMW DNA was sheared into an average fragment size between $12-20 \mathrm{~kb}$ in a Megaruptor 3 system with speed setting 30. Sheared DNA was purified by solid-phase reversible immobilisation using AMPure PB beads with a $1.8 \mathrm{X}$ ratio of beads to sample to remove the shorter fragments and concentrate the DNA sample. The concentration of the sheared and purified DNA was assessed using a Nanodrop spectrophotometer and Qubit Fluorometer and Qubit dsDNA High Sensitivity Assay kit.
Fragment size distribution was evaluated by running the sample on the FemtoPulse system.

\section{Sequencing}

Pacific Biosciences HiFi circular consensus and 10X Genomics read cloud sequencing libraries were constructed according to the manufacturers' instructions. Sequencing was performed by the Scientific Operations core at the Wellcome Sanger Institute on Pacific Biosciences SEQUEL II and Illumina NovaSeq 6000 instruments. Hi-C data were generated from remaining thorax/abdomen tissue using the Arima $\mathrm{Hi}-\mathrm{C}+$ kit and sequenced on a NovaSeq 6000 instrument.

\section{Genome assembly}

Assembly was carried out with Hifiasm (Cheng et al., 2021); haplotypic duplication was identified and removed with 


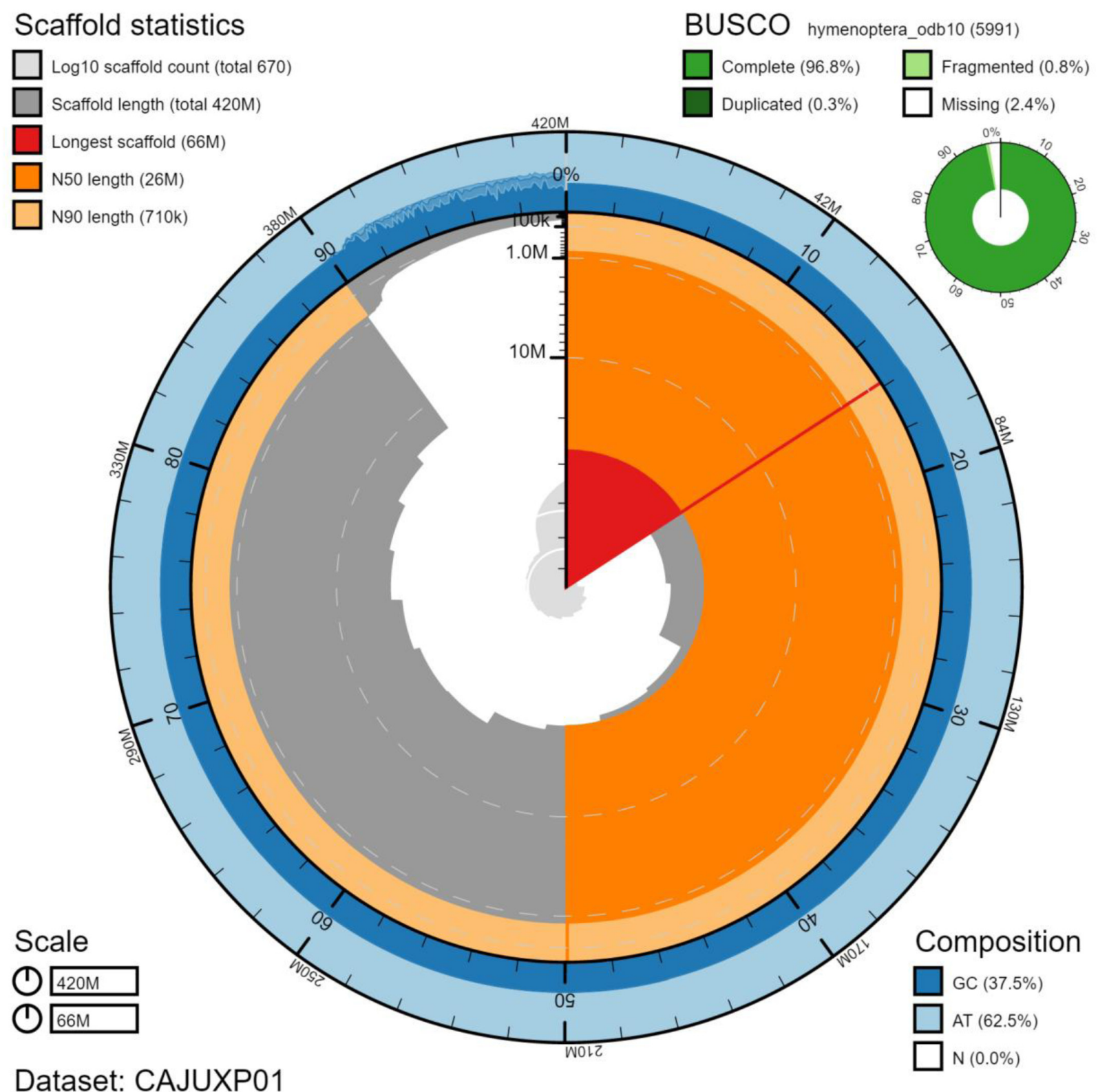

Figure 2. Genome assembly of Cerceris rybyensis, iyCerRyby1.1: metrics. The BlobToolKit Snailplot shows N50 metrics and BUSCO gene completeness. The main plot is divided into 1,000 size-ordered bins around the circumference with each bin representing $0.1 \%$ of the $418,093,278$ bp assembly. The distribution of chromosome lengths is shown in dark grey with the plot radius scaled to the longest chromosome present in the assembly $(66,377,818 \mathrm{bp}$, shown in red). Orange and pale-orange arcs show the N50 and N90 chromosome lengths $(26,175,038$ and 705,187 bp), respectively. The pale grey spiral shows the cumulative chromosome count on a log scale with white scale lines showing successive orders of magnitude. The blue and pale-blue area around the outside of the plot shows the distribution of GC, AT and N percentages in the same bins as the inner plot. A summary of complete, fragmented, duplicated and missing BUSCO genes in the hymenoptera_odb10 set is shown in the top right. An interactive version of this figure is available at https://blobtoolkit.genomehubs. org/view/iyCerRyby1.1/dataset/CAJUXP01/snail.

purge_dups (Guan et al., 2020). One round of polishing was performed by aligning $10 \mathrm{X}$ Genomics read data to the assembly with longranger align, calling variants with freebayes (Garrison \& Marth, 2012). The assembly was then scaffolded with Hi-C data (Rao et al., 2014) using SALSA2 (Ghurye et al., 2019). The assembly was checked for contamination and corrected using the gEVAL system (Chow et al., 2016) as described previously (Howe et al., 2021). 


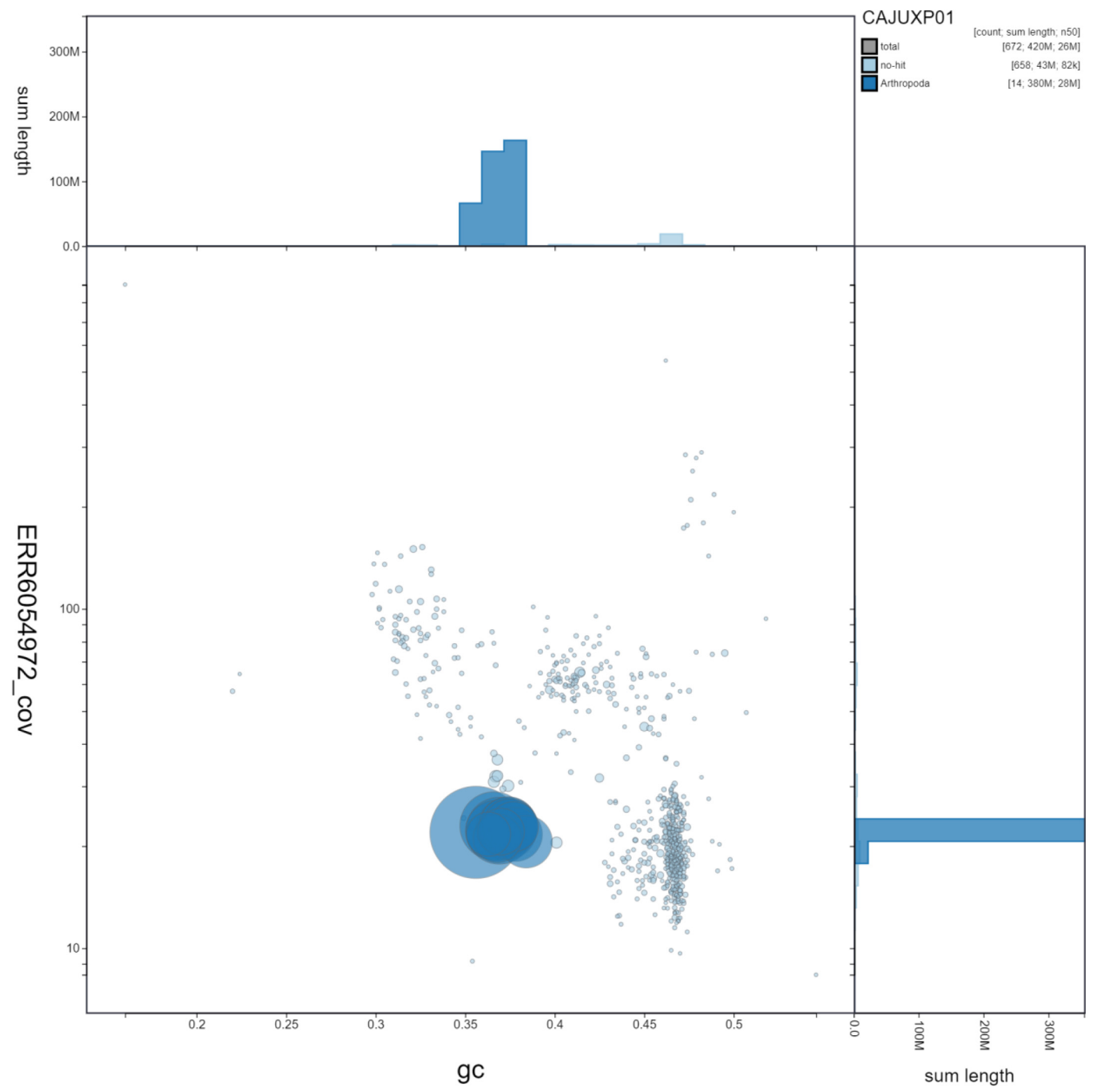

Figure 3. Genome assembly of Cerceris rybyensis, iyCerRyby1.1: GC coverage. BlobToolKit GC-coverage plot. Scaffolds are coloured by phylum. Circles are sized in proportion to scaffold length. Histograms show the distribution of scaffold length sum along each axis. An interactive version of this figure is available at https://blobtoolkit.genomehubs.org/view/iyCerRyby1.1/dataset/CAJUXP01/blob.

Manual curation (Howe et al., 2021) was performed using gEVAL, HiGlass (Kerpedjiev et al., 2018) and Pretext. The mitochondrial genome was assembled using MitoHiFi (Uliano-Silva et al., 2021) and annotated using MitoFinder
(Allio et al., 2020). The genome was analysed and BUSCO scores generated within the BlobToolKit environment (Challis et al., 2020). Table 3 contains a list of all software tool versions used, where appropriate. 


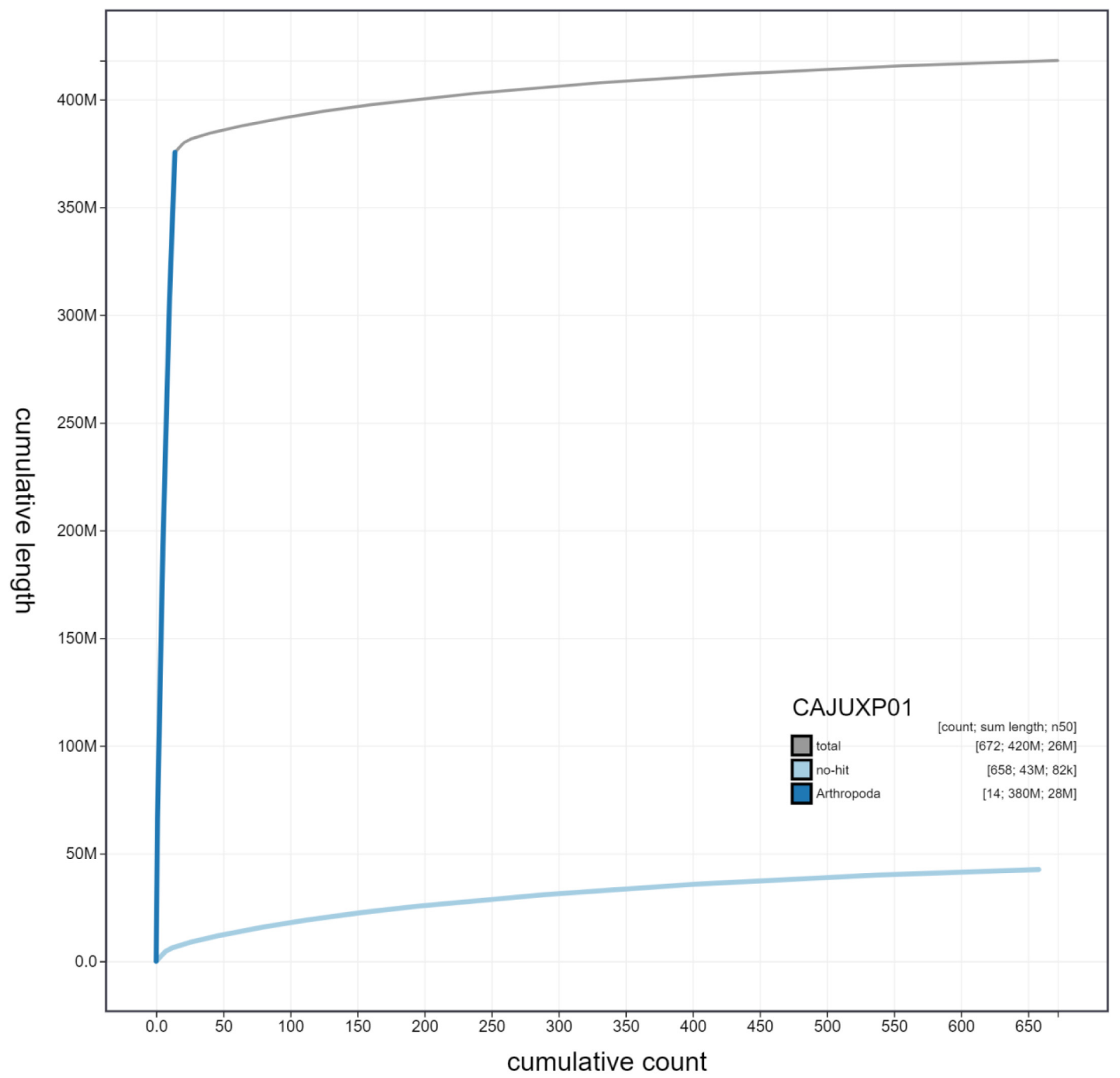

Figure 4. Genome assembly of Cerceris rybyensis, iyCerRyby1.1: cumulative sequence. BlobToolKit cumulative sequence plot. The grey line shows cumulative length for all scaffolds. Coloured lines show cumulative lengths of scaffolds assigned to each phylum using the buscogenes taxrule. An interactive version of this figure is available at https://blobtoolkit.genomehubs.org/view/iyCerRyby1.1/dataset/ CAJUXP01/cumulative.

\section{Ethics/compliance issues}

The materials that have contributed to this genome note have been supplied by a Darwin Tree of Life Partner. The submission of materials by a Darwin Tree of Life Partner is subject to the Darwin Tree of Life Project Sampling Code of Practice.
By agreeing with and signing up to the Sampling Code of Practice, the Darwin Tree of Life Partner agrees they will meet the legal and ethical requirements and standards set out within this document in respect of all samples acquired for, and supplied to, the Darwin Tree of Life Project. Each transfer 


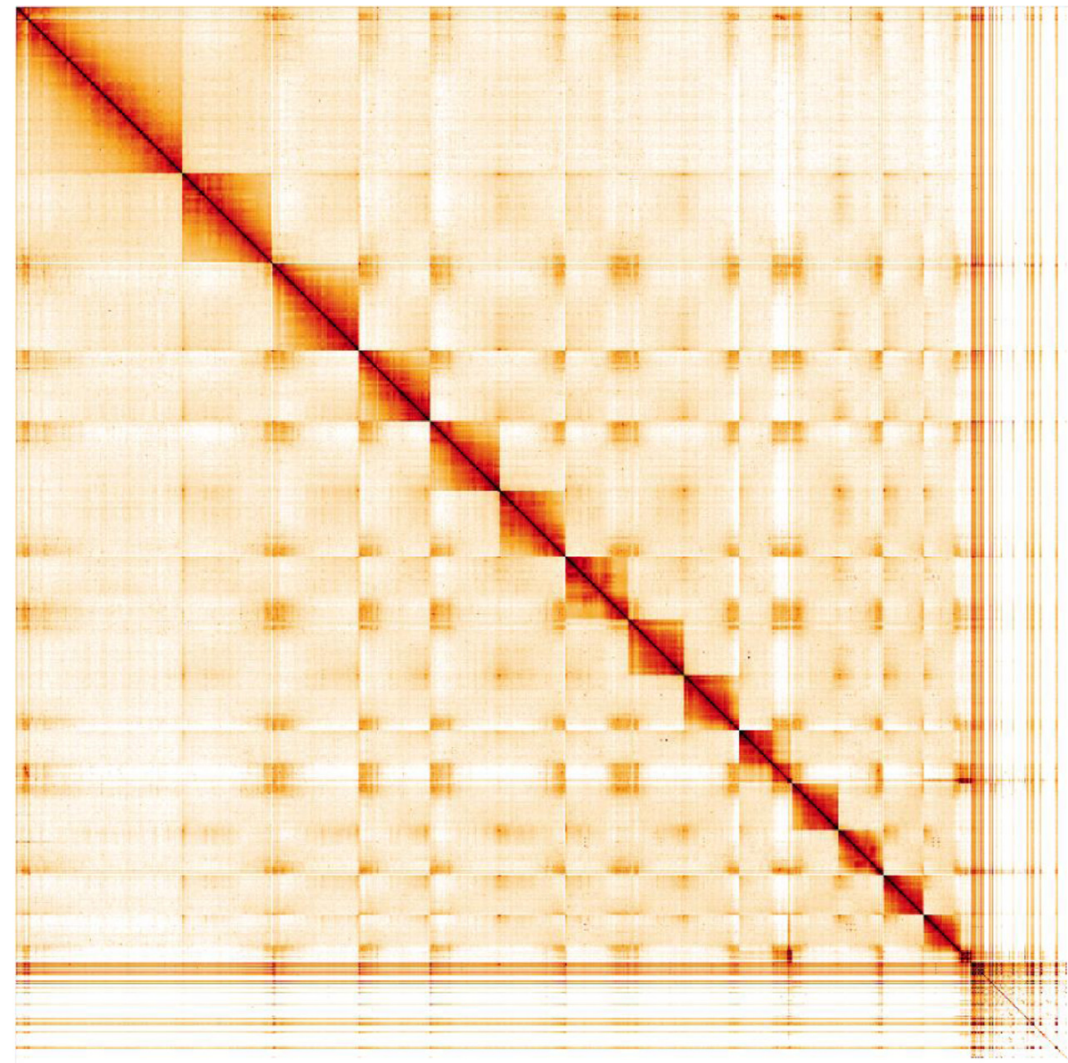

Figure 5. Genome assembly of Cerceris rybyensis, iyCerRyby1.1: Hi-C contact map. Hi-C contact map of the iyCerRyby1.1 assembly, visualised in HiGlass. Chromosomes are shown in order of size from left to right and top to bottom, with unassigned scaffolds shown in the bottom right.

\begin{tabular}{|c|c|c|c|}
\hline INSDC accession & Chromosome & Size (Mb) & GC\% \\
\hline OU342789.1 & 1 & 66.38 & 35.6 \\
\hline OU342790.1 & 2 & 35.56 & 36.6 \\
\hline OU342791.1 & 3 & 34.31 & 36.9 \\
\hline OU342792.1 & 4 & 28.31 & 37.3 \\
\hline OU342793.1 & 5 & 27.62 & 36.7 \\
\hline OU342794.1 & 6 & 26.18 & 37.4 \\
\hline OU342795.1 & 7 & 25.06 & 37.4 \\
\hline OU342796.1 & 8 & 22.18 & 37.5 \\
\hline OU342797.1 & 9 & 21.96 & 37.8 \\
\hline OU342798.1 & 10 & 20.77 & 38.4 \\
\hline OU342799.1 & 11 & 18.65 & 37.7 \\
\hline OU342800.1 & 12 & 17.68 & 36.8 \\
\hline OU342801.1 & 13 & 16.24 & 37.0 \\
\hline OU342802.1 & 14 & 14.61 & 36.3 \\
\hline OU342803.1 & MT & 0.02 & 16.3 \\
\hline - & Unplaced & 42.57 & 43.0 \\
\hline
\end{tabular}


Table 3. Software tools used.

\begin{tabular}{|l|l|l|}
\hline Software tool & Version & Source \\
\hline Hifiasm & 0.15 .1 & Cheng et al., 2021 \\
\hline purge_dups & 1.2 .3 & Guan et al., 2020 \\
\hline SALSA2 & 2.2 & Ghurye et al., 2019 \\
\hline longranger align & 2.2 .2 & $\begin{array}{l}\text { https://support.10xgenomics.com/genome-exome/ } \\
\text { software/pipelines/latest/advanced/other-pipelines }\end{array}$ \\
\hline freebayes & $1.3 .1-17$-gaa2ace8 & Garrison \& Marth, 2012 \\
\hline MitoHiFi & 2 & Uliano-Silva et al., 2021 \\
\hline gEVAL & N/A & Chow et al., 2016 \\
\hline HiGlass & 1.11 .6 & Kerpedjiev et al., 2018 \\
\hline PretextView & $0.2 . x$ & https://github.com/wtsi-hpag/PretextView \\
\hline BlobToolKit & 2.6 .2 & Challis et al., 2020 \\
\hline
\end{tabular}

of samples is further undertaken according to a Research Collaboration Agreement or Material Transfer Agreement entered into by the Darwin Tree of Life Partner, Genome Research Limited (operating as the Wellcome Sanger Institute), and in some circumstances other Darwin Tree of Life collaborators.

\section{Data availability}

European Nucleotide Archive: Cerceris rybyensis (ornate tailed digger wasp). Accession number PRJEB45199; https://www.ebi.ac.uk/ena/browser/view/PRJEB45199.

The genome sequence is released openly for reuse. The C. rybyensis genome sequencing initiative is part of the Darwin Tree of Life (DToL) project. All raw sequence data and the assembly have been deposited in INSDC databases. The genome will be annotated and presented through the Ensembl pipeline at the European Bioinformatics Institute. Raw data and assembly accession identifiers are reported in Table 1.

\section{Author information}

Members of the University of Oxford and Wytham Woods Genome Acquisition Lab are listed here: https://doi.org/10.5281/ zenodo.5746938.

Members of the Darwin Tree of Life Barcoding collective are listed here: https://doi.org/10.5281/zenodo.5744972.

Members of the Wellcome Sanger Institute Tree of Life programme are listed here: https://doi.org/10.5281/zenodo. 5744840

Members of Wellcome Sanger Institute Scientific Operations: DNA Pipelines collective are listed here: https://doi.org/10.5281/ zenodo.5746904.

Members of the Tree of Life Core Informatics collective are listed here: https://doi.org/10.5281/zenodo.5743293.

Members of the Darwin Tree of Life Consortium are listed here: https://doi.org/10.5281/zenodo.5638618.
Allio R, Schomaker-Bastos A, Romiguier J, et al.: MitoFinder: Efficient Automated Large-Scale Extraction of Mitogenomic Data in Target Enrichment Phylogenomics. Mol Ecol Resour. 2020; 20(4): 892-905. PubMed Abstract | Publisher Full Text | Free Full Text

Challis R, Richards E, Rajan J, et al.: BlobToolKit - Interactive Quality Assessment of Genome Assemblies. G3 (Bethesda). 2020; 10(4): 1361-74. PubMed Abstract | Publisher Full Text | Free Full Text

Cheng $\mathrm{H}$, Concepcion GT, Feng X, et al:: Haplotype-Resolved de Novo Assembly Using Phased Assembly Graphs with Hifiasm. Nat Methods. 2021; 18(2): 170-75.

PubMed Abstract | Publisher Full Text | Free Full Text
Chow W, Brugger K, Caccamo M, et al.: gEVAL - a web-based browser for evaluating genome assemblies. Bioinformatics. 2016; 32(16): 2508-10. PubMed Abstract | Publisher Full Text | Free Full Text

Else GR: Cerceris rybyensis (Linnaeus,1771). Bees, Wasp and Ants Recording Society. 1997

Reference Source

Garrison E, Marth G: Haplotype-Based Variant Detection from Short-Read Sequencing. 2012; arXiv: 1207.3907.

Reference Source

Ghurye J, Rhie A, Walenz BP, et al: Integrating Hi-C Links with Assembly Graphs for Chromosome-Scale Assembly. PLoS Comput Biol. 2019; 15(8): 
e1007273.

PubMed Abstract | Publisher Full Text | Free Full Text

Guan D, McCarthy SA, Wood J, et al.: Identifying and Removing Haplotypic Duplication in Primary Genome Assemblies. Bioinformatics. 2020; 36(9): 2896-98.

PubMed Abstract | Publisher Full Text | Free Full Text

Howe K, Chow W, Collins J, et al.: Significantly Improving the Quality of Genome Assemblies through Curation. GigaScience. 2021; 10(1): giaa153. PubMed Abstract | Publisher Full Text | Free Full Text

Kerpedjiev P, Abdennur N, Lekschas F, et al.: HiGlass: Web-Based Visual Exploration and Analysis of Genome Interaction Maps. Genome Biol. 2018; 19(1): 125.

PubMed Abstract | Publisher Full Text | Free Full Text

Kote S, Faktor J, Dapic I, et al: Analysis of Venom Sac Constituents from the Solitary, Aculeate Wasp Cerceris Rybyensis. Toxicon. 2019; 169: 1-4.

PubMed Abstract | Publisher Full Text

Lomholdt: The Sphecidae (Hymenoptera) of Fennoscandia and Denmark.
Fauna Entomologica Scandinavica. 1975; 4: 1-224.

Reference Source

Manni M, Berkeley MR, Seppey M, et al.: BUSCo Update: Novel and Streamlined Workflows along with Broader and Deeper Phylogenetic Coverage for Scoring of Eukaryotic, Prokaryotic, and Viral Genomes. $\mathrm{Mol}$ Biol Evol. 2021; 38(10): 4647-54.

PubMed Abstract | Publisher Full Text | Free Full Text

Rao SSP, Huntley MH, Durand NC, et al:: A 3D Map of the Human Genome at Kilobase Resolution Reveals Principles of Chromatin Looping. Cell. 2014; 159(7): 1665-80.

PubMed Abstract | Publisher Full Text | Free Full Text

Uliano-Silva M, Ferreira Nunes JG, Krasheninnikova K, et al.: marcelauliano/ MitoHiFi: mitohifi_v2.0. 2021.

Publisher Full Text

Zeil J: Orientation Flights of Solitary Wasps (Cerceris; Sphecidae; Hymenoptera). J Comp Physiol A. 1993; 172(2): 207-22.

Publisher Full Text 


\section{Open Peer Review}

\section{Current Peer Review Status:}

\section{Version 1}

Reviewer Report 04 April 2022

https://doi.org/10.21956/wellcomeopenres.19333.r49292

(c) 2022 Menezes R. This is an open access peer review report distributed under the terms of the Creative Commons Attribution License, which permits unrestricted use, distribution, and reproduction in any medium, provided the original work is properly cited.

\section{Rodolpho S. T. Menezes}

Universidade Federal de Santa Maria, Santa Maria, RS, Brazil

This short genome report describes the genome sequencing of the ornate tailed digger wasp, Cerceris rybyensis. The work begins with an introduction to the natural history of the wasp and describes the collection, DNA extraction, sequencing, and assembly. The manuscript is well written. I have only a few minor suggestions:

Abstract:

Please correct the species taxonomy information.

Background:

"Genus"; "genus"

Sequencing:

Why did you use thorax/abdomen tissue and not thorax and legs tissues?

Genome assembly:

Since you assembled the mitochondrial genome, I think it is interesting that you show general (size, GC/AT content...) mitochondrial information about this species.

Is the rationale for creating the dataset(s) clearly described?

Yes

Are the protocols appropriate and is the work technically sound?

Yes

Are sufficient details of methods and materials provided to allow replication by others? Yes

Are the datasets clearly presented in a useable and accessible format? Yes 
Competing Interests: No competing interests were disclosed.

Reviewer Expertise: Phylogenomics, Phylogeography, and Cytogenetics.

I confirm that I have read this submission and believe that I have an appropriate level of expertise to confirm that it is of an acceptable scientific standard.

Reviewer Report 26 January 2022

https://doi.org/10.21956/wellcomeopenres.19333.r47602

(C) 2022 Dearden P. This is an open access peer review report distributed under the terms of the Creative Commons Attribution License, which permits unrestricted use, distribution, and reproduction in any medium, provided the original work is properly cited.

\section{Peter K Dearden}

Genomics Aotearoa, Department of Biochemistry, University of Otago, Dunedin, New Zealand

This manuscript describes the sequencing of the ornate-tailed digger wasp. The work describes the collection, DNA extraction sequencing, assembly, and analysis of the genome, providing all of the necessary metrics. The manuscript is well written, and the figures clear and well explained. Methods are detailed and easy to follow. The sequence produced will be a helpful addition to hymenopteran genomics and the detailed and careful description of the work make this a useful resource for future work.

This work is scientifically sound and I recommend its approval.

Is the rationale for creating the dataset(s) clearly described?

Yes

Are the protocols appropriate and is the work technically sound?

Yes

Are sufficient details of methods and materials provided to allow replication by others? Yes

Are the datasets clearly presented in a useable and accessible format? Yes

Competing Interests: No competing interests were disclosed.

Reviewer Expertise: Genomics

I confirm that I have read this submission and believe that I have an appropriate level of expertise to confirm that it is of an acceptable scientific standard. 